УДК 616.89-008.441.33:612.82

Для цитирования: Асадуллин А.Р., Анцыборов А.В., Ахметова Э.А. Нейровизуализации в клинике расстройств, связанных с употреблением психоактивных веществ (введение в проблему). Сибирский вестник психиатрии и наркологии. 2018; 1 (98): 97-103. https://doi.org/10.26617/1810-3111-2018-1(98)-97-103

\title{
Нейровизуализации в клинике расстройств, связанных с употреблением психоактивных веществ (введение в проблему)
}

\author{
Асадуллин А.P. ${ }^{1,2}$, Анцыборов А.В. ${ }^{3}$, Ахметова Э.А. ${ }^{1,2}$ \\ ${ }^{1}$ Баикирский государственный медицинский университет \\ Башкортостан, 450077, Уфа, ул. Ленина, 3 \\ ${ }^{2}$ Республиканский наркологический диспансер № 1 \\ Башкортостан, 450005, Уфа, ул. Пушкина, 119 \\ ${ }^{3}$ Медициский центр «Альфа Центр Здоровья» \\ Россия, 344022, Ростов-на-Дону, ул. Сочиалистическая, 154 «а»
}

\section{PEЗЮME}

Проведенный анализ литературы, посвященной нейровизуальной картине головного мозга пациентов с наркотической зависимостью, позволяет сделать вывод о том, что практически у всех пациентов имеется дефицит нейрональных связей в отделах мозга, отвечающих за вознаграждение и импульсивность. В настоящем обзоре основное внимание уделено исследованиям, использующим позитронно-эмиссионную томографию (ПЭТ), функциональную магнитно-резонансную томографию (МРТ) и электроэнцефалографию (ЭЭГ) для исследования поведенческих нарушений у пациентов с наркотической зависимостью.

Ключевые слова: дофамин, электроэнцефалография (ЭЭГ), магнитно-резонансная томография (МРТ), позитронноэмиссионная томография (ПЭТ), префронтальная кора.

\section{ВВЕДЕНИЕ}

На сегодняшний день существует широкий спектр методов нейровизуализации процессов и структур головного мозга, которые можно условно разделить на три основные категории: 1) двухфотонные методы визуализации - позитронно-эмиссионная томография (ПЭТ), однофотонные методы визуализации - эмиссионная компьютерная томография (SPECT); 2) магнитно-резонансная томография (МРТ), включающая в себя структурную MPT, функциональную MPT (fMRI) и MPспектроскопию; 3) электрофизиологические методы визуализации - электроэнцефалография (ЭЭГ) и магнитоэнцефалография (МЭГ). Наркотические вещества повышают уровень мезолимбического и мезокортикального дофамина (DA), что способствует формированию зависимости [16, 42]. При длительном употреблении наркотических веществ плотность дофаминовых рецепторов D2 снижается $[59,60,90,94]$. Меняется функционирование дофаминергических рецепторов в кортиколимбической области (с вовлечением орбитофронтальной коры OFC) и передней коры головного мозга (ACC), т.е. отделов, отвечающих за процессы вознаграждения, мотивации и контроля [28, 56, 89].

Цель: анализ литературы, посвященной нейровизуальной картине головного мозга пациентов с наркотической зависимостью

\section{ОБСУЖДЕНИЕ ПРОБЛЕМЫ}

Основные данные нейровизуальных исследований при наркотической зависимости

Интоксикациия

В ситуации острой интоксикации происходит увеличение внеклеточного содержания DA в лимби- ческих областях головного мозга, особенно в области Nucleus accumbens (NAcc). Психостимуляторы, такие как кокаин и метилфенидат (МРН), увеличивают концентрацию DA, блокируя механизмы транспортера дофамина (DAT), как основной механизм рециркуляции DA в нервные окончания. При употреблении «ударных» доз психостимуляторов (например, кокаина) включается механизм, непосредственно связанный с интоксикацией, регулирующий «уровень» блокады DAT [100], как ответ на индукцию ПАВ, увеличивающих содержание DA $[83,99]$. Практически все эффекты повышения концентрации DA напрямую связаны с фармакологическими свойствами кокаина, МРН, амфетамина и других психостимуляторов [29, 45].

ПАВ, относящиеся к так называемым депрессантам (бензодиазепины, барбитураты, алкоголь), лишь косвенно повышают концентрацию DA, во многом за счет воздействия на рецепторный комплекс GABA/бензодиазепин [93, 96]. Опиаты, такие как героин, оксиконт и викодин, оказывают действие путем стимуляции $\mu$-опиоидных рецепторов, из которых некоторые расположены на DA-нейронах и других на GABA-нейронах [103]. Никотин оказывает свои эффекты частично за счет активации ацетилхолиновых никотиновых рецепторов $\alpha 4 \beta 2$, которые также располагаются на DA-нейронах. Никотин, так же как героин и алкоголь, по мнению некоторых авторов, способен влиять на выделение эндогенных опиоидов, что способствует положительным эффектам подкрепления [53]. Марихуана оказывает свои эффекты путем активирующего влияния на каннабиноидные рецепторы первого типа (CB1). 
Последние в свою очередь способны модулировать DA-клетки, а также усиливать ответ постсинаптических нейронов DA [31]. Помимо мезолимбических субкортикальных отделов головного мозга, задействованных при интоксикации ПАВ, префронтальные корковые области (РFC) так же вовлекаются в патофизиологические механизмы интоксикации. Величина повышения концентрации DA уменьшается по мере динамики заболевания - от злоупотребления наркотиками вплоть до сформированной наркотической зависимости [84]. У лиц, употребляющих кокаин, а также в состоянии острой интоксикации кокаином, как и у пациентов с алкоголизмом, отмечается нарушение процессов метаболизма глюкозы в головном мозге [1, 33, 49, 50, 82].

В состоянии острой интоксикации метилфенидатом (МРН) уровень метаболизма глюкозы увеличивается в РFC (префронтальной), ОFС (орбитофронтальной) коре и в стриатуме. Аналогичные результаты получены при исследовании активных потребителей кокаина, у которых также отмечается низкий уровень доступности рецепторов D2 [69, 91, 99]. Употребление кокаина лицами с еще не сформированной зависимостью, наоборот, уменьшает метаболизм глюкозы в префронтальных областях (РFC и OFC) [81]. Исследования состояний наркотической интоксикации с применением FMRI демонстрируют изменения в субкортикальном функционировании стрии после достижения максимальной плазменной концентрации ПАВ [1, 24, 39, 44, 55, 61, $75,80,85]$. Острая интоксикация никотином демонстрировала сильное увеличение активности ЭЭГпоказателей - от низких (дельта, тета, альфа) до высоких (альфа, бета) частот, что является маркером состояния возбуждения $[17,79]$. Низкие дозы алкоголя вызывают изменения в тета- и альфадиапазонах [25]. Эффекты, возникающие на более высоких частотах, как правило, зависят от истории употребления алкоголя и исходных данных ЭЭГ [20, $46,47]$. Рост альфа-активности связан с вызванной употреблением наркотиков эйфорией, «ударных доз» марихуаны [51] или кокаина [36].

Согласно многочисленным исследованиям, в зависимости от стадии кокаиновой зависимости при ЭЭГ-исследовании можно наблюдать увеличение бета- [35, 36], дельта- [35] и альфа-волн в лобных отделах [36], а также процессы увеличения глобальной ЭЭГ-активности [66]. Исследования состояний, связанных с наркотической интоксикацией, с применением различных методик нейровизуализации демонстрируют огромное влияние DA- в PFCобластях, а также влияние на функции стриатума, что количественно определяется увеличением медленных волн ЭЭГ [3, 43].

\section{Крейвинг}

В условиях эксперимента явления крейвинга исследуются показом изображений (видео), содержащих раздражители, связанные с употреблением наркотиков. При использовании данной методики у потребителей кокаина с применением PET и рак- лоприда [11] демонстрировались стимульные видеоролики с кокаином (показ вещества и самого процесса употребления) [97]. У участников эксперимента отмечалось мощное высвобождение DA в дорсальном стриатуме, что напрямую коррелировало с данными самоотчетов потребителей, описывающих явления крейвинга. Наиболее выраженные проявления крейвинга отмечались у лиц с тяжелой зависимостью от кокаина [52, 92, 95].

В другом исследовании с применением РЕТ было показано, что лица с большим стажем наркотизации кокаином могут сохранять определенный уровень когнитивного контроля явлений крейвинга [87, 106]. Результаты исследований показывают наличие связи между рецепторами дофамина D2 в вентральном стриатуме и явлениями мотивации употребления кокаина, что подтверждалось введением раклоприда [11] и десметоксифаллиприда [18, 34, 54].

Области головного мозга, включая амигдалу, гиппокамп и ствол мозга, которые связаны с функциями обработки информации и извлечением ее из памяти, активируются во время усиления явлений крейвинга $[5,11,14,18,31,40]$. Активация данных структур головного мозга также отмечается при синдроме отмены наркотических веществ [23].

Исследования, посвященные изучению изменения метаболизма глюкозы в головном мозге лиц с наркотической зависимостью, подтверждают, что усиление явлений крейвинга у лиц со сформированной зависимостью от наркотиков связано с активацией вентрально-тегментальной области и других мезенцефальных структур [6, 7, 11, 14, 40, 77, 78, 106], медиальных отделов префронтальной корь [28, 77], орбитальньхх отделов фронтальной корь [14, 58, 77, 78, 107], инсулье [18, 26, 74].

При усилении явлений крейвинга активизируются мезокортикальные отделы головного мозга (в том числе орбитальные отделы фронтальной коры). Заинтересованность данных отделов головного мозга отмечается также при ожидании употребления наркотического вещества. У употребляющих кокаин женщин, в отличие от мужчин, исследования при помощи ПЭТ демонстрируют снижение процессов метаболизма в префронтальных областях, связанных с функцией самоконтроля, после стимульных сигналов, демонстрирующих ПАВ (кокаин). По мнению исследователей, данный фактор делает женщин более подверженными рецидиву заболевания [98].

Полученные данные коррелируют с доклиническими исследованиями, демонстрирующими, что уровень эстрогена может увеличивать риск формирования наркотической зависимости у женщин [2]. У пациентов с зависимостью от кокаина в эксперименте регистрировался количественно определяемый высокий бета-ритм и угнетение альфа-ритма [48]. В исследовании зависимых от кокаина пациентов показано усиление бета-ритма наряду с дельтаритмом при использовании стимульных раздражителей, связанных с кокаином (просмотр видеоролика с кокаином). 
Сходные данные были получены в другом исследовании, где сравнивались наркологически здоровые люди и потребители кокаина (употребившие за сутки до эксперимента наркотик) во время состояния отдыха [37, 62]. При никотиновой зависимости в ответ на стимульные раздражители, связанные с сигаретами, наблюдалось усиление тета- и бетаритмов $[38,41,104]$. Приведенные выше результаты различных исследований подтверждают теории, которые описывают зависимость как изменение систем мотивации и вознаграждения головного мозга $[29,70,101]$ и напрямую связаны с явлениями крейвинга $[22,57,105]$. $\Pi A B$

Утрата ситуационного контроля и бинджинг

Нарушение поведенческого самоконтроля возникает во время употребления наркотиков и в состоянии интоксикации, что обусловлено изменениями в области префронтальной коры (ингибирующий эффект в субкортикальной стриарной области) [29]. Исследования с применением методик нейровизуализации показывают, что нейронные субстраты в виде связи отделов таламоорбитофронтальной коры и передней коры головного мозга лежат в основе явлений бинджинга.

У зависимых лиц наблюдается значительное снижение доступности рецепторов D2 в полосатом теле [84], что связано с нарушением процессов метаболизма в области префронтальной коры (особенно в орбитофронтальной коре и дорсолатеральных отделах ПФК). Однако данные нарушения не объясняют полностью поведенческие изменения и нарушения мотивации [27]. Процессы дисрегуляции дофамина в указанных областях головного мозга повышают мотивационную ценность употребления наркотических веществ и зачастую приводят к утрате контроля над потреблением наркотических веществ [29, 101].

В недавно проведенном исследовании с использованием метода fMRI пациентам с зависимостью от кокаина разрешили выбирать, когда и как часто они будут самостоятельно вводить кокаин внутривенно в течение одной контролируемой часовой сессии. Повторяющиеся инъекции наркотика в течение отведенного времени отрицательно коррелировали с активностью в лимбических, паралимбических и мезокортикальных областях, включая оптикофронтальную часть коры головного мозга и лобновисочные отделы. У тех пациентов, кто воздерживался от инъекций наркотика, отмечалась положительная корреляция активности в указанных отделах головного мозга [21, 68].

Процессы искусственного моделирования компульсивного влечения по отношению к нехимическим зависимостям (например, к азартным играм), так же как и исследование возможности контроля над ним, могут сыграть очень важную роль в понимании механизмов утраты контроля при различных зависимостях [27].
Имеющиеся врожденные аномалии в областях островковой доли (insula) и медиальной части префронтальной коры (включая медиальную область орбитофронтальной коры), а также в подкорковых областях (включая стриатум) зачастую связаны с нарушением функции поведенческого контроля, которые отмечаются еще до формирования наркотической зависимости [4]. Результаты цитируемого исследования расширяют привычную нам концепцию формирования наркотической зависимости, выходя за пределы системы вознаграждения [4, 29] и нарушения в системе долговременной памяти [26, 84].

Исследования феномена бинджинга у больных алкоголизмом с применением ЭЭГ выявили преобладание низкочастотного бета-ритма [62]. Бетаактивность может соответствовать количеству и частоте употребления алкоголя [71]. Используя данный признак, возможно инструментально дифференцировать «мало» и «умеренно» пьющих пациентов (коррелируя данные с количеством потребляемого алкоголя) $[19,20]$. Увеличение дельта-ритма наблюдалось у зависимых от алкоголя пациентов с частыми запоями в сравнении со злоупотребляющими алкоголем пациентами и малопьющими людьми [64]. Тета- и альфа-ритмы одновременно увеличивались по частоте спустя 25 минут после употребления алкоголя в привычных для испытуемых дозах [67]. В основе бинджинга лежат изменения в дофаминергической, серотонинергической и глутаматергической системах организма [12].

Синдром отмены и реиидив

Исследования синдрома отмены у потребителей кокаина с применением методики ПЭТ демонстрируют снижение метаболизма глюкозы в области префронтальной коры в первые 10 дней лишения наркотика в сравнении с контрольной группой [85, 86]. В другом исследовании после ночного воздержания употребления и замены никотина на жвачку у обследуемых отмечалось снижение МРТ-сигнала в области таламуса во время лишения никотина. При этом МРТ-сигнал усиливался в области вентрального стриатума, когда происходило замещение никотина [76]. При исследовании метаболизма глюкозы во время синдрома отмены алкоголя отмечалось снижение метаболической активности в области стриато-таламо-орбитофронтальной коры. Аналогичные изменения отмечались и на этапе детоксикации $[10,86,100]$. У пациентов с зависимостью от кокаина сообщается о схожих метаболических изменениях в области вентрального стриатума [102]. При процедуре детоксикации большая метаболическая активность отмечалась в орбитофронтальной коре и базальных ганглиях, особенно на ранних этапах синдрома отмены (1-я неделя абстиненции) [86]. Низкая метаболическая активность имела место в области префронтальной коры в период от 1-й до 6-й недели после последнего употребления наркотика [82]. 
Низкий показатель связывания D2 дофаминовых рецепторов в области стрии во время абстинентного синдрома отмечался у потребителей кокаина [99], алкоголя [100], героина [103], метамфетамина [84], а также у курильщиков со стажем, но в период лишения сигарет [22]. Замедление метаболической активности исключительно в области орбитофронтальной коры наблюдалось у зависимых от метамфетамина лиц [81]. Данное обстоятельство делает систему вознаграждения менее чувствительной к естественным наградам [9] и другим нехимическим вознаграждениям (например, деньгам) [28].

Индуцированные наркотическим веществом изменения в функциях префронтальной коры и орбитофронтальной коры (а также стрии и инсулы) ухудшают способность моделировать эмоции, что имеет важное значение для преодоления стрессовых ситуаций [65]. Многими исследователями отмечается, что этот фактор является мощным предиктором в развитии рецидива заболевания [73].

Исследования абстинентного периода вследствие употребления кокаина с использованием ЭЭГ демонстрирует уменьшение дельта- [12,37] и тетаволн $[13,37]$. При этом отмечается увеличение альфа- и бета-активности [37]. В другом исследовании описано увеличение альфа-активности на раннем этапе развития абстинентного синдрома у пациентов с зависимостью от героина [72]. В отличие от ЭЭГкартины, наблюдаемой при кокаиновой абстиненции, при синдроме отмены никотина происходит усиление тета-активности на фоне снижения альфаи бета-активности [17, 79]. Снижение альфаактивности, по мнению некоторых авторов, напрямую связано с длительностью абстинентного периода. Показатель альфа-активности может выступать в роли своеобразной «меры измерения» острых эффектов отмены ПАВ [30].

Анализ ERP во время синдрома отмены у пациентов с алкоголизмом представил увеличение латентности амплитуд N200 и P300 наряду с уменьшение амплитуд N100 и P300 [64]. Уменьшение амплитуды Р300 явилось частым признаком во время абстинентного периода у зависимых от кокаина [30], героина [63], а также у лиц с никотиновой абстиненцией $[6,8,15]$. Показатели ЭЭГ и ERP предлагается использовать для прогнозирования возможного рецидива. Так, альфа- и тета-активность у трезвых алкоголиков различались с точностью 83-85\% в сравнении с абстинентами и теми, у кого произошел рецидив заболевания [88].

Повышенная активация ЦНС, определяемая по высокой бета-активности, по мнению некоторых исследователей, является надежным маркером между абстинентами и теми, у кого произошел рецидив заболевания [71]. Исследования ERP у трезвых алкоголиков демонстрировали задержку периода латентности N200, что позволяет провести различие между абстинентами и теми, у кого произошел рецидив, с общей достоверностью прогноза до $71 \%$ [32]. Уменьшение амплитуды Р300 при выполнении
ERP можно использовать в качестве фактора достоверного прогноза рецидива (до $71 \%$ ) у пациентов с зависимостью от кокаина [7].

\section{ВЫВОДЫ}

Исследования в области нейровизуализации помогают лучше понять сложные процессы головного мозга, формирующиеся в результате употребления наркотических веществ. Нейровизуальные маркеры помогают практикующим врачам в возможности прогнозирования рецидива заболевания и в ряде случаев могут играть решающую роль в разработке новых методик лечения.

\section{КОНФЛИКТ ИНТЕРЕСОВ}

Авторы заявляют об отсутствии конфликта интересов в связи с публикацией данной статьи.

\section{ИСТОЧНИК ФИНАНСИРОВАНИЯ}

Авторы заявляют об отсутствии финансирования при проведении исследования.

\section{ЛИТЕРАTУPA/REFERENCES}

1. Adams K.M. et al. The significance of family history status in relation to neuropsychological test performance and cerebral glucose metabolism studied with positron emission tomography in older alcoholic patients. Alcoholism: Clinical and Experimental Research. 1998; 22 (1): 105-110.

2. Anker J.J., Carroll M.E. Females are more vulnerable to drug abuse than males: evidence from preclinical studies and the role of ovarian hormones. Biological Basis of Sex Differences in Psychopharmacology. Springer Berlin Heidelberg, 2010: 73-96.

3. Bassareo V., De Luca M. A., Di Chiara G. Differential expression of motivational stimulus properties by dopamine in nucleus accumbens shell versus core and prefrontal cortex. Journal of neuroscience. 2002; 22 (11): 4709-4719.

4. Bechara A. Decision making, impulse control and loss of willpower to resist drugs: a neurocognitive perspective. Nature neuroscience. 2005; 8 (11): 1458-1463.

5. Bonson K.R. et al. Neural systems and cue-induced cocaine craving. Neuropsychopharmacology. 2002. 26: 376-386. doi:10.1016/S0893-133X(01)00371-3712

6. Brody A.L. et al. Attenuation of cue-induced cigarette craving and anterior cingulate cortex activation in bupropion-treated smokers: a preliminary study. Psychiatry Research: Neuroimaging. 2004; 130 (3): 269-281.

7. Brody A. L. et al. Brain metabolic changes during cigarette craving. Archives of General Psychiatry. 2002; 59 (12): 11621172 .

8. Buckley P. Association of Low Striatal Dopamine D2 Receptor Availability with Nicotine Dependence Similar to That Seen with Other Drugs of Abuse. Year Book of Psychiatry \& Applied Mental Health. 2009; 2009: 324.

9. Cassens G. et al. Amphetamine withdrawal: effects on threshold of intracranial reinforcement. Psychopharmacology. 1981; 73 (4): $318-322$.

10. Catafau A.M. et al. Regional cerebral blood flow changes in chronic alcoholic patients induced by naltrexone challenge during detoxification. Journal of Nuclear Medicine. 1999; 40 (1): 19.

11. Childress A.R. et al. Limbic activation during cue-induced cocaine craving. American Journal of Psychiatry. 1999; 156 (1): $11-18$.

12. Cornish J.L., Duffy P., Kalivas P.W. A role for nucleus accumbens glutamate transmission in the relapse to cocaine-seeking behavior. Neuroscience. 1999; 93 (4): 1359-1367.

13. Courtney K.E., Polich J. Binge drinking effects on EEG in young adult humans. International journal of environmental research and public health. 2010; 7 (5): 2325-2336.

14. Daglish M.R.C. et al. Functional connectivity analysis of the neural circuits of opiate craving): "more" rather than "different"? Neuroimage. 2003; 20 (4): 1964-1970.

15. Daurignac E. et al. 522 Attentional withdrawal and smoking cessation): A longitudinal ERP study. International Journal of Psychophysiology. 1998; 30 (1-2): 201-202. 
16. Di Chiara G. A motivational learning hypothesis of the role of mesolimbic dopamine in compulsive drug use. Journal of psychopharmacology. 1998; 12 (1): 54-67.

17. Domino E.F. Effects of tobacco smoking on electroencephalographic, auditory evoked and event related potentials. Brain and cognition. 2003; 53 (1): 66-74.

18. Due D. L. et al. Activation in mesolimbic and visuospatial neural circuits elicited by smoking cues): evidence from functional magnetic resonance imaging. American Journal of Psychiatry. 2002; 159 (6): 954-960.

19. Ehlers C.L., Schuckit M.A. EEG fast frequency activity in the sons of alcoholics. Biological psychiatry. 1990; 27 (6): 631-641.

20. Ehlers C.L., Wall T.L., Schuckit M.A. EEG spectral characteristics following ethanol administration in young men. Electroencephalography and Clinical Neurophysiology. 1989; 73 (3): 179-187.

21. Foltin R.W. et al. The effects of escalating doses of smoked cocaine in humans. Drug and Acohol Dependence. 2003; 70 (2): 149-157.

22. Franken I.H.A. Drug craving and addiction): integrating psychological and neuropsychopharmacological approaches. Progress in Neuro-Psychopharmacology and Biological Psychiatry. 2003; 27 (4): 563-579.

23. Franklin T.R. et al. Limbic activation to cigarette smoking cues independent of nicotine withdrawal): a perfusion fMRI study. Neuropsychopharmacology. 2007; 32 (11): 2301-2309.

24. Gilman J.M. et al. Why we like to drink): a functional magnetic resonance imaging study of the rewarding and anxiolytic effects of alcohol. Journal of Neuroscience. 2008; 28 (18): 4583-4591.

25. Glenn S.W., Sinha R., Parsons O.A. Electrophysiological indices predict resumption of drinking in sober alcoholics. Alcohol. 1993; 10 (2): 89-95.

26. Goldstein R.Z. et al. Anterior cingulate cortex hypoactivations to an emotionally salient task in cocaine addiction. Proceedings of the National Academy of Sciences. 2009; 106 (23): 9453-9458.

27. Goldstein R.Z. et al. Dopaminergic response to drug words in cocaine addiction. Journal of Neuroscience. 2009; 29 (18): 6001-6006.

28. Goldstein R.Z. et al. Role of the anterior cingulate and medial orbitofrontal cortex in processing drug cues in cocaine addiction. Neuroscience. 2007; 144 (4): 1153-1159.

29. Goldstein R. Z., Volkow N. D. Drug addiction and its underlying neurobiological basis): neuroimaging evidence for the involvement of the frontal cortex. American Journal of Psychiatry. 2002; 159 (10): 1642-1652.

30. Gooding D. C., Burroughs S., Boutros N. N. Attentional deficits in cocaine-dependent patients): converging behavioral and electrophysiological evidence. Psychiatry Research. 2008; 160 (2): 145-154.

31. Grant S. et al. Activation of memory circuits during cue-elicited cocaine craving. Proceedings of the National Academy of Sciences. 1996; 93 (21): 12040-12045.

32. Grüsser S. M. et al. Cue-induced activation of the striatum and medial prefrontal cortex is associated with subsequent relapse in abstinent alcoholics. Psychopharmacology. 2004; 175 (3): 296302.

33. Gu H. et al. Mesocorticolimbic circuits are impaired in chronic cocaine users as demonstrated by resting-state functional connectivity. Neuroimage. 2010; 53 (2): 593-601.

34. Heinz A., Siessmeier T., Wrase J. Correlation Between Dopamine D Receptors in the Ventral Striatum and Central Processing of Alcohol Cues and Craving. Year Book of Psychiatry \& Applied Mental Health. 2006; 2006): 81-82.

35. Herning R.I. et al. Cocaine increases EEG beta): a replication and extension of Hans Berger's historic experiments. Electroencephalography and Clinical Neurophysiology. 1985; 60 (6): $470-477$.

36. Herning R.I. et al. Cocaine-induced increases in EEG alpha and beta activity): evidence for reduced cortical processing. Neuropsychopharmacology. 1994; 11 (1): 1-9.

37. Herning R.I. et al. Neurophysiological signs of cocaine dependence): increased electroencephalogram beta during with drawal. Biological Psychiatry. 1997; 41 (11): 1087-1094.
38. Herrmann M.J. et al. Event related potentials and cue reactivity in alcoholism. Alcoholism: Clinical and Experimental Research. 2000; 24 (11): 1724-1729.

39. Ingvar M. et al. Alcohol activates the cerebral reward system in man. Journal of Studies on Alcohol. 1998; 59 (3): 258-269.

40. Kilts C.D. et al. Neural activity related to drug craving in cocaine addiction. Archives of General Psychiatry. 2001; 58 (4): 334-341.

41. Knott V. et al. EEG correlates of imagery-induced cigarette craving in male and female smokers. Addictive Behaviors. 2008; 33 (4): 616-621.

42. Koob G.F. et al. Role for the mesocortical dopamine system in the motivating effects of cocaine. NIDA research monograph. 1994; 145: 1-1.

43. Koob G.F., Volkow N.D. Neurocircuitry of addiction. Neuropsychopharmacology. 2010; 35 (1): 217-238.

44. Kufahl P.R. et al. Neural responses to acute cocaine administration in the human brain detected by fMRI. Neuroimage. 2005; 28 (4): 904-914.

45. Laruelle M. et al. SPECT imaging of striatal dopamine release after amphetamine challenge. Journal of Nuclear Medicine. 1995; 36 (7): 1182-1190.

46. Lehtinen I. et al. Individual alcohol reaction profiles. Alcohol. 1985; 2 (3): 511-513.

47. Lehtinen I., Lang A.H., Keskinen E. Acute effect of small doses of alcohol on the NSD parameters (normalized slope descriptors) of human EEG. Psychopharmacology. 1978; 60 (1): 87-92.

48. Liu X. et al. Effect of cocaine-related environmental stimuli on the spontaneous electroencephalogram in polydrug abusers. Neuropsychopharmacology. 1998; 19 (1): 10-17.

49. London E.D. et al. Cocaine-induced redoppuction of glucose utilization in human brain): A study using positron emission tomography and [fluorine 18]-fluorodeoxyglucose. Archives of General Psychiatry. 1990; 47 (6): 567-574.

50. London E.D. et al. Morphine-induced metabolic changes in human brain): studies with positron emission tomography and [fluorine 18] fluorodeoxyglucose. Archives of General Psychiatry. 1990; 47 (1): 73-81.

51. Lukas S.E., Mendelson J.H., Benedikt R. Electroencephalographic correlates of marihuana-induced euphoria. Drug and Alcohol Dependence. 1995; 37 (2): 131-140.

52. Maas L.C. et al. Functional magnetic resonance imaging of human brain activation during cue-induced cocaine craving. American Journal of Psychiatry. 1998; 155 (1): 124-126.

53. Mansvelder H.D., McGehee D.S. Long-term potentiation of excitatory inputs to brain reward areas by nicotine. Neuron. 2000; 27 (2): 349-357.

54. Martinez D. et al. Alcohol dependence is associated with blunted dopamine transmission in the ventral striatum. Biological Psychiatry. 2005; 58 (10): 779-786.

55. Mathew R.J. et al. Regional cerebral blood flow after marijuana smoking. Journal of Cerebral Blood Flow \& Metabolism. 1992; 12 (5): 750-758.

56. McClure S.M., York M.K., Montague P.R. The neural substrates of reward processing in humans): the modern role of FMRI. The Neuroscientist. 2004; 10 (3): 260-268.

57. Mogg K. et al. Eye movements to smoking $\square$ related pictures in smokers): relationship between attentional biases and implicit and explicit measures of stimulus valence. Addiction. 2003; 98 (6): 825-836.

58. Myrick H. et al. Differential brain activity in alcoholics and social drinkers to alcohol cues): relationship to craving. Neuropsychopharmacology. 2004; 29 (2): 393.

59. Nader M.A. et al. PET imaging of dopamine D2 receptors during chronic cocaine self-administration in monkeys. Nature Neuroscience. 2006; 9 (8).

60. Nader M.A., Czoty P.W. PET imaging of dopamine D2 receptors in monkey models of cocaine abuse): genetic predisposition versus environmental modulation. American Journal of Psychiatry. 2005; 162 (8): 1473-1482.

61. Nakamura H. et al. Activation of fronto-limbic system in the human brain by cigarette smoking): evaluated by a CBF measurement. The Keio Journal of Medicine. 2000; 49: A122-4. 
62. Noldy N.E. et al. Quantitative EEG changes in cocaine withdrawal): Evidence for long-term CNS effects. Neuropsychobiology. 1994; 30 (4): 189-196.

63. Papageorgiou C.C. et al. Long-term abstinence syndrome in heroin addicts): indices of P300 alterations associated with a short memory task. Progress in Neuro-Psychopharmacology and Biological Psychiatry. 2004; 28 (7): 1109-1115.

64. Parsons O.A., Sinha R., Williams H.L. Relationships between Neuropsychological Test Performance and Event Related Potentials in Alcoholic and Nonalcoholic Samples. Alcoholism: Clinical and Experimental Research. 1990; 14 (5): 746-755.

65. Payer D.E. et al. Differences in cortical activity between methamphetamine-dependent and healthy individuals performing a facial affect matching task. Drug and Alcohol Dependence. 2008; 93 (1): 93-102.

66. Reid M.S. et al. Cocaine cue versus cocaine dosing in humans): evidence for distinct neurophysiological response profiles. Pharmacology Biochemistry and Behavior. 2008; 91 (1): 155164.

67. Reid M.S. et al. Topographic imaging of quantitative EEG in response to smoked cocaine self-administration in humans. Neuropsychopharmacology. 2006; 31 (4): 872-884.

68. Risinger R.C. et al. Neural correlates of high and craving during cocaine self-administration using BOLD fMRI. Neuroimage. 2005; 26 (4): 1097-1108.

69. Ritz M.C. et al. Cocaine receptors on dopamine transporters are related to self-administration of cocaine. Science. 1987; 237: 1219-1224.

70. Robinson T.E., Berridge K.C. Incentive sensitization and addiction. Addiction. 2001; 96 (1): 103-114.

71. Saletu-Zyhlarz G.M. et al. Differences in brain function between relapsing and abstaining alcohol-dependent patients, evaluated by EEG mapping. Alcohol and Alcoholism. 2004; 39 (3): 233 240 .

72. Shufman E. et al. Electro-encephalography spectral analysis of heroin addicts compared with abstainers and normal controls. The Israel Journal of Psychiatry and Related Sciences. 1996; 33 (3): 196-206.

73. Sinha R. et al. Imaging stress-and cue-induced drug and alcohol craving): association with relapse and clinical implications. Drug and Alcohol Review. 2007; 26 (1): 25-31.

74. Smolka M.N. et al. Severity of nicotine dependence modulates cue-induced brain activity in regions involved in motor preparation and imagery. Psychopharmacology. 2006; 184 (3-4): 577 588.

75. Stein E.A. et al. Nicotine-induced limbic cortical activation in the human brain): a functional MRI study. American Journal of Psychiatry. 1998; 155 (8): 1009-1015.

76. Tanabe J. et al. Ventral striatal blood flow is altered by acute nicotine but not withdrawal from nicotine. Neuropsychopharmacology. 2008; 33 (3): 627-633.

77. Tapert S.F. et al. fMRI BOLD response to alcohol stimuli in alcohol dependent young women. Addictive Behaviors. 2004; 29 (1): 33-50.

78. Tapert S.F. et al. Neural response to alcohol stimuli in adolescents with alcohol use disorder. Archives of General Psychiatry. 2003; 60 (7): 727-735.

79. Teneggi V. et al. EEG power spectra and auditory P300 during free smoking and enforced smoking abstinence. Pharmacology Biochemistry and Behavior. 2004; 77 (1): 103-109.

80. Tiihonen J. et al. Acute ethanol-induced changes in cerebral blood flow. American Journal of Psychiatry. 1994; 151 (10): $1505-1508$.

81. Volkow N.D. et al. Activation of orbital and medial prefrontal cortex by methylphenidate in cocaine-addicted subjects but not in controls): relevance to addiction. Journal of Neuroscience. 2005; 25 (15): 3932-3939.

82. Volkow N.D. et al. Acute effects of ethanol on regional brain glucose metabolism and transport. Psychiatry Research: Neuroimaging. 1990; 35 (1): 39-48.

83. Volkow N.D. et al. Association of methylphenidate-induced craving with changes in right striato-orbitofrontal metabolism in cocaine abusers): implications in addiction. American Journal of Psychiatry. 1999; 156 (1): 19-26.
84. Volkow N.D. et al. Brain DA D2 receptors predict reinforcing effects of stimulants in humans): replication study. Synapse. 2002; 46 (2): 79-82.

85. Volkow N.D. et al. Cerebral blood flow in chronic cocaine users): a study with positron emission tomography. The British Journal of Psychiatry. 1988; 152 (5): 641-648.

86. Volkow N.D. et al. Changes in brain glucose metabolism in cocaine dependence and withdrawal. The American Journal of Psychiatry. 1991; 148 (5): 621.

87. Volkow N.D. et al. Cognitive control of drug craving inhibits brain reward regions in cocaine abusers. Neuroimage. 2010; 49 (3): 2536-2543.

88. Volkow N.D. et al. Decreased brain metabolism in neurologically intact healthy alcoholics. The American Journal of Psychiatry. 1992; 149 (8): 1016

89. Volkow N.D. et al. Decreased dopamine D2 receptor availability is associated with reduced frontal metabolism in cocaine abusers. Synapse. 1993; 14 (2): 169-177.

90. Volkow N.D. et al. Decreased striatal dopaminergic responsiveness in detoxified cocaine-dependent subjects. Nature. 1997; 386 (6627): 830-833.

91. Volkow N.D. et al. Decreases in dopamine receptors but not in dopamine transporters in alcoholics. Alcoholism: Clinical and Experimental Research. 1996; 20 (9): 1594-1598.

92. Volkow N.D. et al. Dopamine increases in striatum do not elicit craving in cocaine abusers unless they are coupled with cocaine cues. Neuroimage. 2008; 39 (3): 1266-1273.

93. Volkow N.D. et al. Effects of acute alcohol intoxication on cerebral blood flow measured with PET. Psychiatry Research. 1988; 24 (2): 201-209.

94. Volkow N.D. et al. Effects of chronic cocaine abuse on postsynaptic dopamine receptors. Am J Psychiatry. 1990; 147 (6): 719724.

95. Volkow N.D. et al. High levels of dopamine D2 receptors in unaffected members of alcoholic families): possible protective factors. Archives of General Psychiatry. 2006; 63 (9): 999-1008.

96. Volkow N.D. et al. Imaging dopamine's role in drug abuse and addiction. Neuropharmacology. 2009; 56: 3-8.

97. Volkow N.D. et al. Imaging endogenous dopamine competition with [11C] raclopride in the human brain. Synapse. 1994; 16 (4): $255-262$

98. Volkow N.D. et al. Reduced metabolism in brain "control networks" following cocaine-cues exposure in female cocaine abusers. PloS one. 2011; 6 (2): e16573.

99. Volkow N.D. et al. Reinforcing effects of psychostimulants in humans are associated with increases in brain dopamine and occupancy of D2 receptors. Journal of Pharmacology and Experimental Therapeutics. 1999; 291 (1): 409-415.

100. Volkow N.D. et al. Relationship between subjective effects of cocaine and dopamine transporter occupancy. Nature. 1997; 386 (6627): 827-830

101. Volkow N.D., Fowler J.S. Addiction, a disease of compulsion and drive): involvement of the orbitofrontal cortex. Cerebral Cortex. 2000; 10 (3): 318-325.

102. Volkow N.D., Fowler J.S., Wang G.J. Imaging studies on the role of dopamine in cocaine reinforcement and addiction in humans. Journal of Psychopharmacology. 1999; 13 (4): 337-345.

103. Wang G.J. et al. Dopamine D2 receptor availability in opiatedependent subjects before and after naloxone-precipitated withdrawal. Neuropsychopharmacology. 1997; 16 (2): 174-182.

104. Warren C.A., McDonough B.E. Event-related brain potentials as indicators of smoking cue-reactivity. Clinical Neurophysiology. 1999; 110 (9): 1570-1584.

105. Waters A.J. et al. Attentional shifts to smoking cues in smokers. Addiction. 2003; 98 (10): 1409-1417.

106. Wexler B.E. et al. Functional magnetic resonance imaging of cocaine craving. American Journal of Psychiatry. 2001; 158 (1): 86-95.

107. Wrase J. et al. Development of alcohol-associated cues and cueinduced brain activation in alcoholics. European Psychiatry. 2002; 17 (5): 287-291.

Поступила в редакцию 3.11.2017 Утверждена к печати 5.02.2018 
Асадуллин Азат Раилевич, психиатр-нарколог, к.м.н., доцент кафедры психиатрии и наркологии с курсом ИДПО. Анцыборов Андрей Викторович, психиатр-нарколог.

Ахметова Эльвина Аслямовна, психиатр-нарколог, ассистент кафедры психиатрии и наркологии с курсом ИДПО.

Ахметова Эльвина Аслямовна, aea1202@yandex.ru

УДК 616.89-008.441.33:612.82

For citation): Asadullin A.R., Antsyborov A.V., Akhmetova E.A. Neuroimaging of the clinical picture of substance use disorders (Introduction to the problem). Siberian Herald of Psychiatry and Addiction Psychiatry. 2018; 1 (98): 97-103. https://doi.org/10.26617/1810-3111-2018-1(98)-97-103

\section{Neuroimaging of the clinical picture of substance use disorders (Introduction to the problem)}

Asadullin A.R. ${ }^{1,2}$, Antsyborov A.V. ${ }^{3}$, Akhmetova E.A. ${ }^{1,2}$

${ }^{1}$ Bashkir State Medical University Lenin Street 3, 450077, Ufa, Bashkortostan

${ }^{2}$ Republican Narcological Dispensary no. 1 Pushkin Street 119, 450005, Ufa, Bashkortostan

${ }^{3}$ Medical Center "Alpha Health Center"

Socialisticheskaya Street 154 “a”, 344022, Rostov-on Don, Russian Federation

\section{ABSTRACT}

The analysis of the literature on the neuroimaging of the cerebral brain of patients with drug dependence suggests that virtually all patients have a deficit of neural connections, in the brain responsible for remuneration and impulsivity. This review focuses on studies using positron emission tomography (PET), functional magnetic resonance imaging (MRI), and electroencephalography (EEG) to investigate behavioral disorders in patients with drug dependence. tex.

Keywords: dopamine; electroencephalography; magnetic resonance imaging; positron emission tomography; prefrontal cor-

Asadullin Azat R., psychiatrist-expert in narcology, PhD, senior lecturer of the Department of Psychiatry and Narcology with course of IAPE, Bashkir State Medical University; Republican Narcological Dispensary no. 1, Ufa, Bashkortostan.

Antsyborov Andrey V., psychiatrist-expert in narcology, Medical Center "Alpha Health Center", Rostov-on Don, Russian Federation

Akhmetova Elvina A., psychiatrist-expert in narcology, assistant to the Department of Psychiatry and Narcology with course of IAPE, Bashkir State Medical University; Republican Narcological Dispensary no. 1, Ufa, Bashkortostan.

Akhmetova Elvina A., aea1202@yandex.ru 